\title{
INTERACTIVE TOOLS FOR LINEAR ALGEBRA: GEOUNIUD
}

\author{
Maria Antonietta Lepellere \\ University of Udine, Italy \\ Francesco Zucconi \\ University of Udine, Italy \\ Nizar Salahi Al Asbahi \\ University of Udine, Italy
}

\author{
Alberto Carminati \\ University of Udine, Italy
}

\begin{abstract}
GeoUniud is a user-friendly platform built-in interactive tutors which allow students to investigate specific tasks by selecting their own input values and working through a problem in a step-by-step fashion together with immediate feedback at each step. Lessons and exercises are stored and organized with a careful use of randomized controlled contents as exercises, geometrical pictures and abstract reasoning. The lessons are augmented by a virtually infinite collection of examples, and by interactive representations of concepts. As example we show the design of two interactive tools about linear transformation and change of basis in order to develop students' sense-making in a dynamic geometry environment (DGE) within the perspective of semiotic mediation.
\end{abstract}

Keywords: dynamic geometry environment, linear algebra, semiotic mediation, technology.

\section{Introduction}

The Bologna Declaration and the creation of the European Higher Education Area (European Commission, 2009) have promoted a structural change in the European universities. This change, which has been increasingly addressed in recent years, has not been limited to a mere restructuring of the academic curriculum but has served to change the paradigm of university teaching. In this new scenario, the advance of constructivist methods in teaching practices seems to have become the hegemonic method. Some of the characteristics of such methods that are currently being imposed in general, not only within the field of mathematics, are as follow: student-focused teaching; an increase in participation by the students themselves, favouring discussion between students and instructors and departing from the traditional method of teaching through lectures; the use of educational technology. 
But integration of technology into existing pedagogy is not easy and requires careful thought as to the redesign of classroom instruction. Advocates of technology use note that technology tools should "serve as intellectual partners during activities requiring problem solving or critical thinking" (Ertmer \& Ottenbreit-Leftwich, 2013, 176). As online learning requires the use of technology to access and interact with instructors and materials, the design and implementation of online instruction brings new and more complex issues to light. Research in mathematics education has long highlighted the complex and systemic nature of mathematics teaching/learning situations, which requires, even in the case of e-learning, a specificity in the production and organization of the learning environment (Albano \& Ferrari, 2008).

The theory of semiotic mediation (TSM), proposed by Bartolini Bussi \& Mariotti (2008), considers that in a mathematics class, when using an artefact for accomplishing a mathematical task, students can be led to produce personal signs which can be put in relationship with mathematical signs. The construction of such relationship should be assumed as an explicit educational aim by the teacher, who can intentionally orient her/his own action towards promoting the evolution of signs expressing the relationship between the artefact and the tasks into signs expressing the relationship between the artefact and the knowledge at stake. The semiotic potential of an artefact consists of a twofold relationship “... (1) between a tool and meanings emerging in the accomplishment of the task and (2) between the tool and meanings related to specific mathematical content evoked by that use and recognizable by an expert” (Mariotti, 2014, p. 157).

About dynamic geometry environment (DGE) Leung, Chan \& Lopez-Real, 2006 write: "A key feature of DGE is its ability to visually represent geometrical invariants amidst simultaneous variations induced by dragging activities. This dynamic tool - dragging - induces potential dialectic between the conceptual realm (abstraction) of mathematical entities and the world of virtual empirical objects. Because of this possibility, dragging has been a major focus of research in DGE resulting in fruitful discussions on promising dragging modalities and strategies that seem to be conducive to knowledge construction”.

\section{Literature review}

Problems in teaching and learning of linear algebra have a long history in many countries (Dorier \& Sierpinska, 2001). Frequently, the abstract character and the formalism of mathematics that students have not been exposed to in school before is named as a central obstacle (a variety of studies are outlined and evaluated in Dorier, Robert, Robinet, \& Rogalski, 2000). In addition to these general reasons of conceptual difficulty, some researchers (Dorier et al., 2000; Hillel, 2000) reveal the reasons for the difficulties that are specific to linear 
algebra. One reason is that the course on linear algebra includes many concepts that students have limited (if any) experiences in, such as vector space, subspace, span, linear independence, basis, and dimension (Dorier et al., 2000; Hillel, 2000). Another issue is that students in linear algebra courses conducted at the university level (Hillel, 2000) are required to think and question these concepts and their related procedures not only in specific situations $\left(\mathrm{R}^{2}, \mathrm{R}^{3}, 2 \mathrm{x} 2\right.$, $3 \times 3$ type matrices, etc.) but also in general situations (V vector space, algebraic structures, etc.). This situation causes students to make high-level abstractions while struggling to understand many new concepts for them.

In a linear algebra course, linear transformations are introduced via algebraic rules and are usually associated with matrices, and matrix multiplication, as they appear in many textbooks, and after this, a number of geometric applications follow, such as reflection and rotation (Kolman \& Hill, 2007). Therefore, the existing knowledge of undergraduate students concerning functions is often neglected, and linear transformations, on the one hand, are introduced as ready-made mathematics, and, on the other hand, applications of the topic are introduced in a static way. Research results from the related literature show that students are not fully aware of the mathematical relationship between the notions of function and linear transformation (Bagley, Rasmussen, \& Zandieh, 2015). This situation leads to the following question: How may we best facilitate students' connection of the notions of function, matrix and transformation matrix? In this paper, the focus is on a dynamic view, and interactive tools are presented with the aim of introducing students to geometric transformations and their relationship with function; specifically, with linear functions and their matrix representation.

Several studies emphasized the use of DGE for the visualization, especially in Geometer's Sketchpad (Gol Tabaghi, 2014; Caglayan, 2015) and GeoGebra (Beltrán-Meneu, Murillo-Arcila, \& Albarracin, 2016; Turgut, 2019). Cooley et al. (2014) availed themselves of the affordances of GeoGebra to aid students' visualization of the ways in which points on polygons are transformed. Turgut (2019) presents a careful analysis of students' use of the dragging tool in GeoGebra to make sense of transformations matrix, using a lens of semiotic mediation. Another study (Martin, Loch, Cooley, Dexter, \& Vidakovic, 2010) used software such as Photoshop or GIMP for image manipulation to support students' visualization of linear transformations. Dominiquez-Garcia, GarciaPlana, \& Taberna (2016) highlighted the use of MatLab as a tool for teaching linear algebra to engineers through mathematical modelling. Many of these studies leverage existing software to support visualization efforts. In contrast, Dogan (2018) developed an interactive web-based module to visualize vectors geometrically in $\mathrm{R}^{3}$. 
Anyone using a DGE may experience dynamic visualisation, based on perceiving variation through dragging, which may contribute to the user making a conjecture about the geometric properties of the figures (Mariotti, 2014). Following this idea, we can hypothesize that specific tools and functions of a DGE, may be exploited to foster the transition from function to a matrix representation of geometric transformation in $\mathrm{R}^{2}$.

In this research, a TSM perspective having two interrelated components is considered. On the one hand, the TSM is specific to the integration of digital tools in the teaching and learning of mathematics. On the other hand, an elaboration of the semiotic potential of an artefact guides an instructor by providing a possible learning route regarding didactic goals. We design interactive tools in order to develop students' sense-making regarding matrix representation of geometric transformations in a DGE within the perspective of semiotic mediation. In particular, the focus is on students' reasoning on the transition from the notion of function to transformation and to matrix representation of geometric transformations in $\mathrm{R}^{2}$ and $\mathrm{R}^{3}$. Along these lines, the theory of semiotic mediation is referred to as a theoretical framework in the design of a teaching and learning environment for the emergence of mathematical thinking. Innovative digital materials will be created in relation to the needs of single course teachers and taking into account the difficulties of the students documented both by currently research studies and teacher experience.

\section{Methodology}

The proposed tools fit into the context of asynchronous and blended learning, moving from a teacher-centered approach to a student-centered approach. The teacher therefore has the most complex task of guiding and facilitating. The aim is to promote the widening of the "horizontal" dimension of student preparation, putting at his disposal innovative didactic material supported by self-evaluation tools, in familiar format for students. The contents will be structured by degrees of difficulty that will progressively prepare the user not only to solve exercises but also to produce well-constructed formal proofs. The material is suitable for use in a flipped learning classroom.

The platform, under the name GeoUniud, was organized according to a modular structure, to guarantee maximum flexibility and accessibility. Naturally, it was implemented in order to guarantee compatibility with the University's current e-learning Moodle platform. It is neither economically nor culturally productive to try to replace the teacher's function with a virtual copy of it, inevitably destined to a rigid as well as rapid obsolescence. In fact, one of the objectives of the project is to support teachers without usurping their role, but rather assist them in the creation of innovative content and thus freeing them 
from the heavy commitment of acquiring technical skills often not relevant to their assignment, yet requires a modern teaching. GeoUniud has the possibility to integrate the work "in vertical mode", carried out in class through a frontal lesson, with work done independently, in "horizontal mode", controlled indirectly by the teacher by means of adaptive self-regulation criteria entered in the platform.

We construct interactive tools in order to strengthen student sense-making regarding matrix representation of geometric transformations in a DGE environment within the perspective of semiotic mediation. In particular, the focus is on helping the students' reasoning on the transition from the notion of function to transformation and to matrix representation of geometric transformations in $\mathrm{R}^{2}$ and $\mathrm{R}^{3}$. Along these lines, the theory of semiotic mediation is used as a theoretical structure in the design of the teaching and learning environment to bring out the mathematical thinking of students.

When producing materials for a class, the tool chosen comes with some unavoidable compromises: the notation available can be slightly different from the one used by the teacher and the students, the range and quality of examples and exercises could be narrow and lacking, or suitable only for a small part of the whole course. This can result in choosing different tools during different learning periods and requires some commitment from students and teacher alike to adapt and reach some level of proficiency. Moreover, a narrow selection of tools or a limited experience in bending them to more creative uses can shape courses and teaching styles. A prime example of this last dynamic may in our opinion be MOOC courses: widely participated and "only online”, they are built around three kind of resources, i.e. video lessons, static html/pdf pages and quizzes, and present a distinct teaching style. These tools can be very useful, but they surely can't be the most suitable for every subject, nor reach the heights promised by the latest technological innovations.

Speaking of more complex and innovative tools, well established software available today come from an era where an application was self-sufficient and somewhat rigid and are adapting quickly to a new reality where flexibility and accessibility are of utmost importance. GeoGebra offers its "GeoGebra materials" for the classroom, with a wide range of applications a teacher can arrange and release to students during a course, but all these applications are separated and often written by different developers with different aims.

Maple established a new language to write applications and Maplesoft, the software house behind it, sells products and services written with it. But as of today, we could not find a tool, be it a language or an application, that offers the means to build a course with a consistent quota of interactive elements with consistent quality, and readily adaptable to different teachers and subjects. Premium offers of ad hoc development are pricy and impose at least a basic 
rigid notation and some limitations, and support can depend on the future commercial success of specific languages or firms.

The natural solution is to start from scratch, choosing languages that grant accessibility and are nowhere at risk of being abandoned, i.e. html/JavaScript. Lot of resources are available that are maintained by universities and open source communities, and this means they are already learning-oriented and free from the stiffness that an already well-developed software can have. Writing in $\mathrm{html} / \mathrm{JavaScript}$ forces one to circumvent some obstacles but is rewarding in that a random generated example fits perfectly in the flow of a page, without forcing the reader to open a different window. The pages with exercises offer all the perks of a self-standing application, but can be hosted anywhere, from a simple amateurish server to a university's Moodle platform, without requiring special setups or plugins not without security implications. What's more important, GeoUniud let students flow seamlessly from theoretical to training pages, interacting all the way with random generated examples and building a record of their performances. This record lets the platform adapt the difficulty of other training pages, unlock new exercises or even entire modules of the course.

\section{Research results}

GeoUniud has a number of user-friendly built-in interactive tutors which allow students to investigate specific tasks by selecting their own input values and working through a problem in a step-by-step fashion together with immediate feedback at each step. Students can avoid having to use specific syntax by using this point-and-click interface allowing them to concentrate more specifically on a given problem. The performance at each step requires the student to make a cognitive decision where the thinking process reinforces the concept involved.

The students are not fully aware of the mathematical relationship between the notions of function and linear transformation and we may facilitate students' connection of the notions of function, matrix and transformation matrix with a dynamic view, interactive tools with the aim of introducing students to geometric transformations and their relationship with function; specifically, with linear functions and their matrix representation. For other interactive tools, concerning systems of linear equations, see Lepellere et al. (2020).

Exercise1: The student should specify coefficients for the matrix representation of a linear transformation from $\mathrm{R}^{2}$, where the vector space's basis is known, to $\mathrm{R}^{3}$, with the canonical basis (Figure 1). The exercise shows the two spaces with representations of a vector element and its image, but any explicit expression of the linear map is omitted. 
SOCIETY. INTEGRATION. EDUCATION

Proceedings of the International Scientific Conference. Volume VI, May $22^{\text {th }}-23^{\text {th }}, 2020.678-688$

Exercise 1

Consider a linear map $f: \mathbb{R}^{2} \rightarrow \mathbb{R}^{3}$. In the leftmost graphic you can see a generic vector $v$ belonging to the domain of $f$, while the rightmost graphic shows its image $f(v)$. Dragging $v$ and seeing how $f(v)$ changes, find the coefficients of $M_{\mathcal{E}, B}(f)$, where

$$
\mathcal{B}=\left\{\left(\begin{array}{c}
2 \\
-1
\end{array}\right),\left(\begin{array}{l}
3 \\
2
\end{array}\right)\right\}
$$

and insert them in the empty fields below.

In the $3 D$ graphic, the following colors are associated to the 3 axes: $x$-axis- $y$-axis- $z$-axis

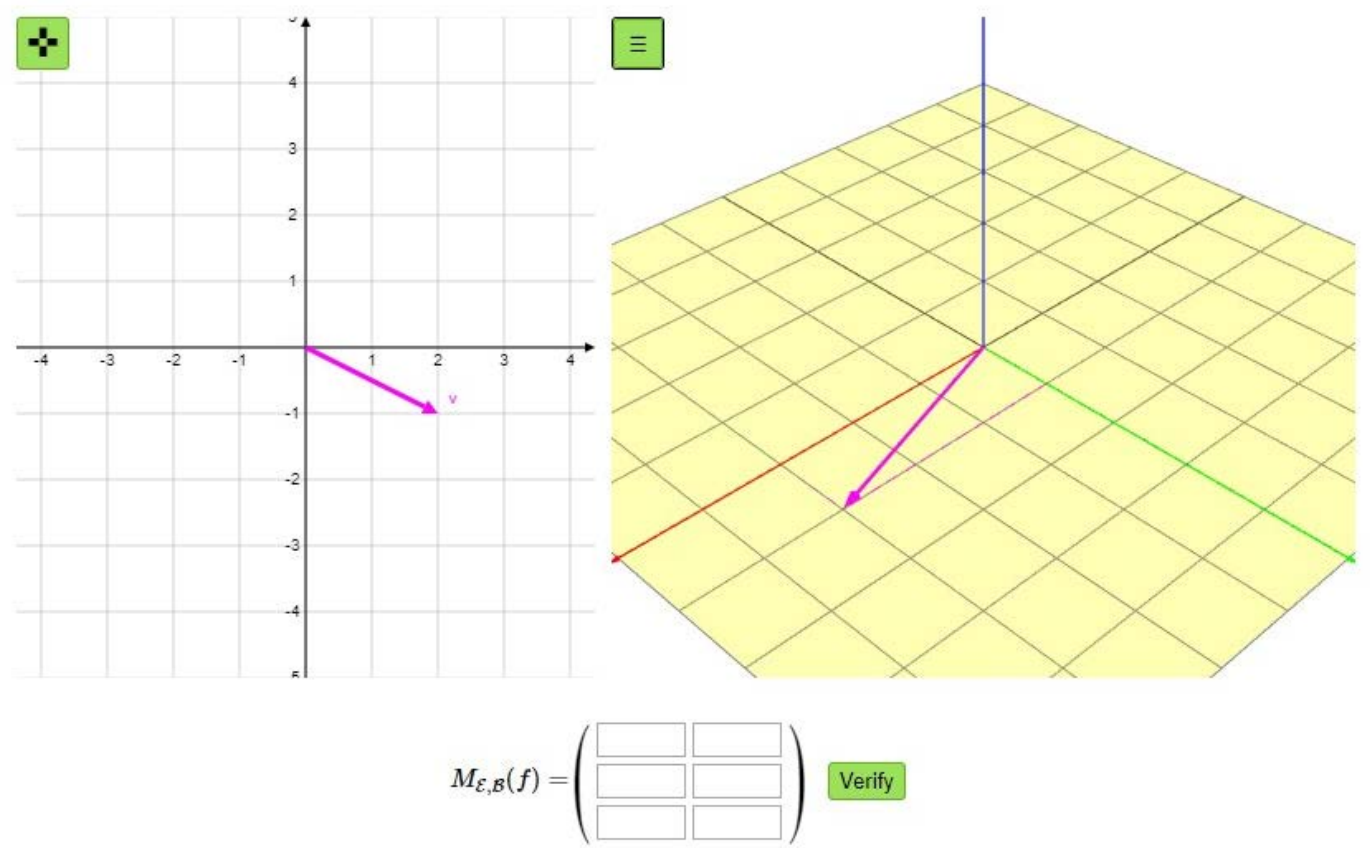

Figure 1 Exercise 1

The student can manipulate the bidimensional vector by dragging and observe in the parallel dynamic three-dimensional space the image through the linear map (Figure 2), enriched by projections and other visual helpers to make depth perception easier. If the student is well prepared for this activity, he/she can aim at finding the images of the two basis vectors to determine the requested transformation matrix and input the coefficients in the specific grid of fields.
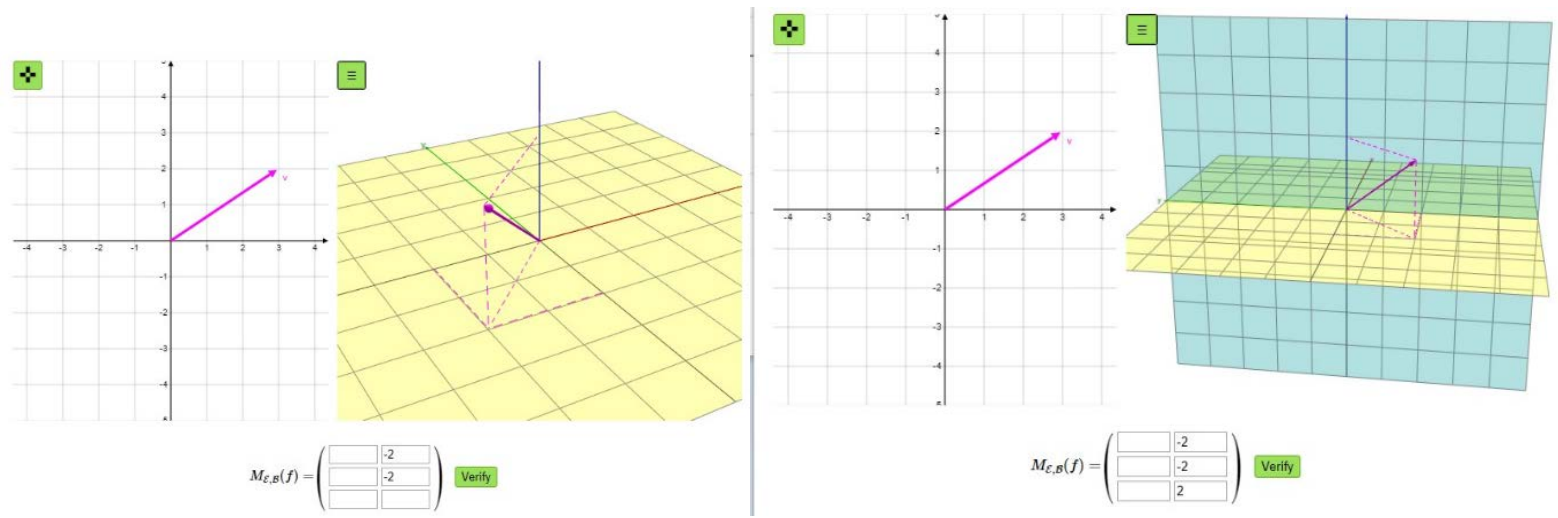

Figure 2 The image of the linear map 
When the fields are filled and the result is submitted, the page gives feedback with eventually simple messages, when not all the fields are filled or the input themselves are of an invalid nature (Figure 3), or give a colorimetric feedback, filling the grid with green where the result is the right one, or red where it is incorrect.

$$
M_{\mathcal{E}, \mathcal{B}}(f)=\left(\begin{array}{|l|l|}
\hline 3 & -2 \\
\hline & -2 \\
\hline & 2
\end{array}\right) \text { Verify } \quad M_{\mathcal{E}, \mathcal{B}}(f)=\left(\begin{array}{|l|l|}
\hline 3 & -2 \\
\hline 1 & -2 \\
\hline z & 2 \\
\hline 2
\end{array}\right) \text { Verify }
$$

Figure 3 Feedback

Exercise 2. The student must determine the coefficients of the matrices of transition from a basis $\mathrm{B}$ to a basis $\mathrm{C}$ and vice versa. To find the values of the various coefficients the student can help himself with a 2D whiteboard (Figure 4). By using the "Basis represented" input field and the "canonical"/"basis" buttons, the student can see the following types of visualization:

\section{Exercise 2}

Dragging the vector $v$ on the graphic below, determine both change of basis matrices $M_{B, \mathcal{C}}$ and $M_{\mathcal{C}, B}$, where

$$
\mathcal{B}=\left\{\left(\begin{array}{c}
1 \\
-1
\end{array}\right),\left(\begin{array}{l}
2 \\
1
\end{array}\right)\right\}, \quad \mathcal{C}=\left\{\left(\begin{array}{l}
3 \\
0
\end{array}\right),\left(\begin{array}{c}
-2 \\
2
\end{array}\right)\right\}
$$

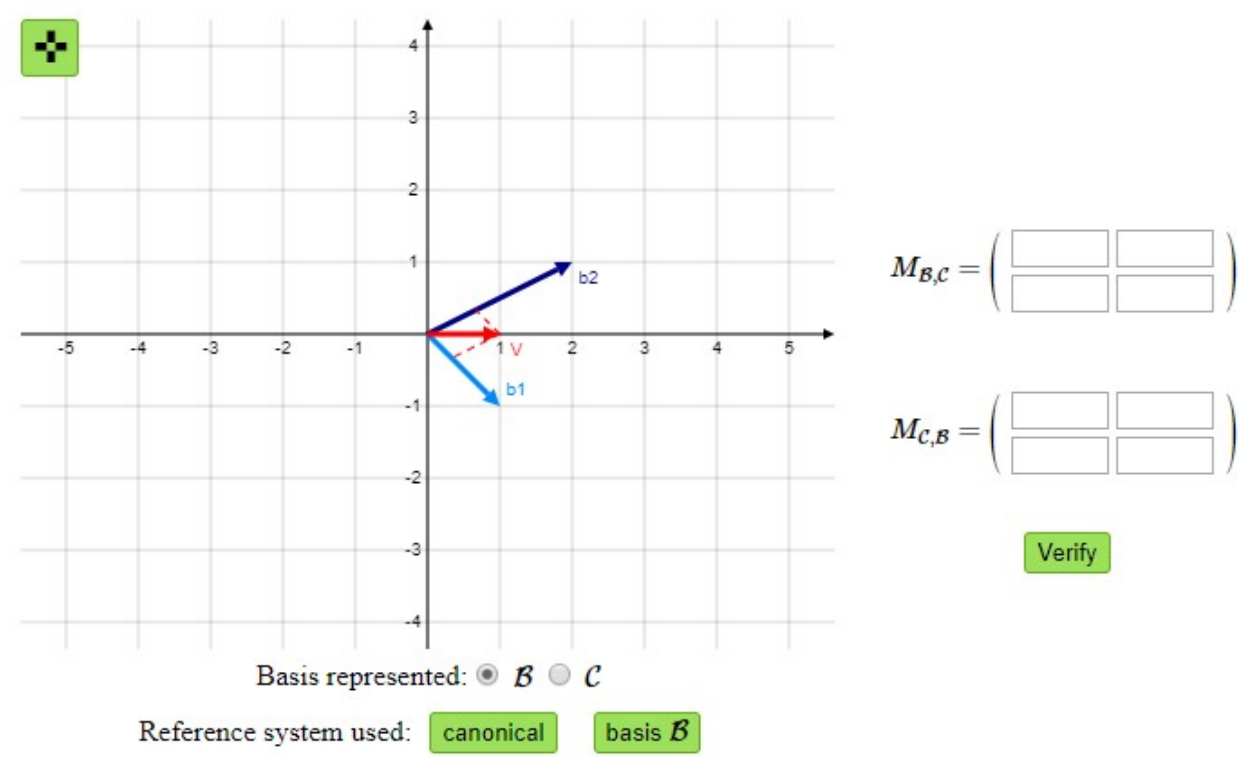

Figure 4 Exercise 2 
1) Vectors of basis $B$ and canonical reference system (used to set the red vector so that it matches $\mathrm{C}_{1}$ or $\mathrm{C}_{2}$ ).

2) Reference system of basis $B$ (used to display the coordinates of the red vector with respect to basis $\mathrm{B}$ ).

3) Vectors of basis $C$ and canonical reference system (used to set the red vector so that it matches $b_{1}$ or $b_{2}$ ).

4) Reference system of basis $C$ (used to display the coordinates of the red vector with respect to basis $C$ ).
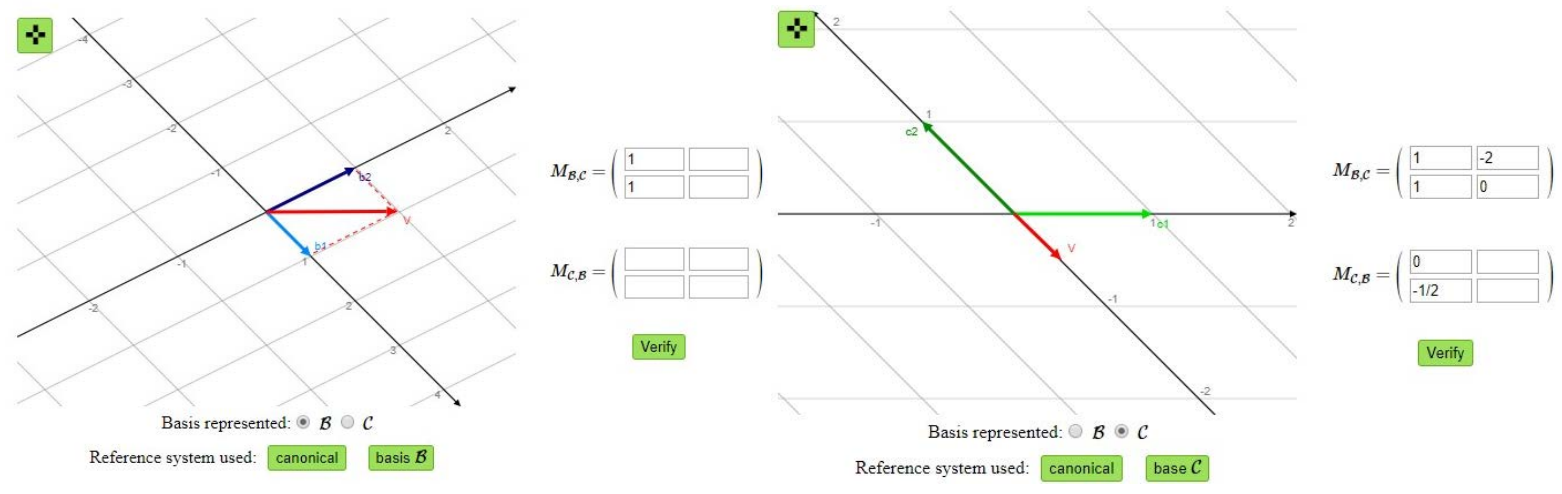

Figure 5 On the left step 2: the red vector represents $c_{1}$ and its projections show its coordinates with respect to basis $B$. On the right step 4: the red vector represents $b_{1}$ and its projections show its coordinates with respect to basis $C$

As before, at the end of the exercise, the boxes are highlighted in green or in red to indicate the right and wrong values (Figure 6). The platform also provides feedback if some boxes had been left empty or had non-numeric values

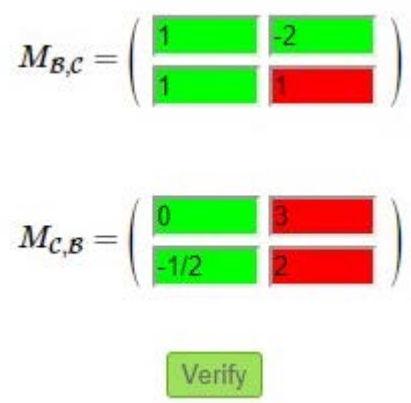

Figure 6 Feedbacks

\section{Conclusion and discussion}

GeoUniud is a user-friendly platform, where lessons and exercises are stored and organized with a careful use of randomized controlled contents as exercises, geometrical pictures and abstract reasoning. The lessons are 
augmented by a virtually infinite collection of examples, and by interactive representations of concepts.

In this paper we have described two interactive tools; one is designed to enhance the understanding of the interplay between the concept of function, linear transformation and its matrix representation. The other is on the mutual relation between the process of change of basis and its matrix presentation. The creation of a specific DGE environment provides a context for students' sensemaking on matrix representation of geometric transformation and change of basis. However, this designed context can be considered as a heuristic tool for the preparation of students to enter abstract vector spaces. Moreover, as emphasized in the TSM, classroom discussion dynamics play an effective role in the transformation of personal meanings into mathematical meanings. Interpersonal exchanges in classroom discussion may be helpful to students in the construction of a bridge between the output linearity of equations and representing them as matrices or other interplays.

\section{References}

Albano, G., \& Ferrari, P.L. (2008). Integrating technology and research in mathematics education: the case of e-learning. In Garcia Penalvo, F.J. (Ed.), Advances in E-learning: Experiences and Methodologies (pp. 132-148). Information Science Reference (IGI Global), Hershey (PA-USA).

Bagley, S., Rasmussen, C., \& Zandieh, M. (2015). Inverse, composition, and identity: the case of function and linear transformation. The Journal of Mathematical Behavior, 37, 3647.

Beltran-Meneu, M.J., Murillo-Arcila, M., \& Albarracin, L. (2016). Emphasizing visualization and physical applications in the study of eigenvectors and eigenvalues. Teaching Mathematics and Its Applications: An International Journal of the IMA, 36(3), 123-135.

Bartolini Bussi, M.G., \& Mariotti, M.A. (2008). Semiotic mediation in the mathematics classroom: Artifacts and signs after a Vygotskian perspective. In L. English, Bartolini Bussi, M., Jones, G., Lesh, R., \& Tirosh, D. (Eds.), Handbook of international research in mathematics education (Vol. 2, pp. 746-783). Mahwah: Erlbaum.

Caglayan, G. (2015). Making sense of eigenvalue-eigenvector relationships: Math majors' linear algebra-geometry connections in a dynamic environment. The Journal of Mathematical Behavior, 40, 131-153.

Cooley, L., Vidakovic, D., Martin, W.O., Dexter, S., Suzuki, J., \& Loch, S. (2014). Modules as learning tools in linear algebra. PRIMUS, 24(3), 257-278.

Dogan, H. (2018). Differing instructional modalities and cognitive structures: Linear algebra. Linear Algebra and Its Applications, 542, 464-483.

Dominguez-Garcia, S., Garcia-Plana, M.I., \& Taberna, J. (2016). Mathematical modelling in engineering: An alternative way to teach linear algebra. International Journal of Mathematical Education in Science and Technology, 47(7), 1076-1086.

Dorier, J.L., \& Sierpinska, A. (2001). Research into the teaching and learning of linear algebra'. In D. Holton (Ed.), The Teaching and Learning of Mathematics at University 
SOCIETY. INTEGRATION. EDUCATION

Proceedings of the International Scientific Conference. Volume VI, May $22^{\text {th }}-23^{\text {th }}, 2020.678-688$

Level (pp. 255-274). An ICMI Study, Kluwer Academic Publishers, Dortrecht/Boston/London.

Dorier, J.L., Robert, A., Robinet, J., \& Rogalski, M. (2000). The obstacle of formalism in linear algebra. In Dorier, J.L. (Ed.), On the teaching of linear algebra (pp. 85-124). Dordrecht, the Netherlands: Kluwer Academic Publishers.

Ertmer, P.A., \& Ottenbreit-Leftwich, A. (2013). Removing obstacles to the pedagogical changes required by Jonassen's vision of authentic technology-enabled learning. Computers \& Education, 64, 175-182.

European Commission. (2009). Directorate-General for Education and Cultures. ECTS users' guide. Retrieved from http://ec.europa.eu/education/tools/docs/ects-guide_en.pdf

Gol Tabaghi, S. (2014). How dragging changes students' awareness: Developing meanings for eigenvector and eigenvalue. Canadian Journal of Science, Mathematics and Technology Education, 14(3), 223-237.

Hillel, J. (2000). Modes of description and the problem of representation in linear algebra. In J.L. Dorier (Ed.), On the Teaching of Linear Algebra (pp. 91-207). New York: Springer.

Lepellere, M.A., Zucconi, F., Salahi Al Asbahi, N., Carminati, A. (2020). MatUniud: Tools for Linear Algebra, INTED2020 Proceedings, In Press.

Leung, A., Chan, Y.C., \& Lopez-Real, F. (2006). Instrumental genesis in dynamic geometry environments. In L.H. Son, N. Sinclair, J.B. Lagrange, \& C. Hoyles (Eds.), Proceedings of the ICMI 17 Study Conference: Part 2, (pp. 346-353).

Mariotti, M.A. (2014). Transforming images in a DGS: The semiotic potential of the dragging tool for introducing the notion of conditional statement. In S. Rezat, M. Hattermann \& A. Peter-Koop (Eds.), Transformation a fundamental idea of mathematics education (pp. 155-172). New York: Springer.

Martin, W., Loch, S., Cooley, L., Dexter, S., \& Vidakovic, D. (2010). Integrating learning theories and application-based modules in teaching linear algebra. Linear Algebra and Its Applications, 432(8), 2089-2099.

Kolman, B., \& Hill, D. (1999). Linear Algebra with Applications. New York: Kluwer Academic Publishers.

Turgut, M. (2019). Sense-making regarding matrix representation of geometric transformations in $\mathrm{R}^{2}$ : a semiotic mediation perspective in a dynamic geometry environment. $Z D M$, 1-16. 